\section{La montée en puissance}

des lasers et l'accroissement

de la finesse des cavités

optiques permettent aujourd'hui

le développement de sources

de rayons $X$ fondées sur

la diffusion Compton inverse.

Ces sources compactes

fourniront des faisceaux

de haute intensité, accordables

en énergie dans la gamme

20-100 keV, utilisables dans

divers domaines : biomédecine,

préservation de l'héritage

culturel, science des matériaux

notamment.

Elles fonctionneront dans un

environnement d'exploitation

plus facile d'accès.

Des méthodes expérimentales utilisées aujourd'hui

uniquement auprès des sources

de rayonnement synchrotron

pourront être adoptées dans

le cadre d'un laboratoire,

d'un hôpital ou d'un musée.

ThomX est le projet français

dans ce domaine.

\title{
ThomX : un démonstrateur de source compacte de rayons $X$
}

Christelle Bruni $^{(1)}$, Marie Jacquet ${ }^{(1)}$, Alexandre Loulergue ${ }^{(2)}$,

Hugues Monard $^{(1)}$ (monard@lal.in2p3.fr) et Fabian Zomer ${ }^{(1)}$

(1) Laboratoire de I'Accélérateur Linéaire, Université Paris-Sud et IN2P3/CNRS, 91898 0rsay Cedex

(2) Synchrotron SOLEIL, L'Orme des Merisiers, Saint-Aubin, BP 48, 91192 Gif-sur-Yvette Cedex

Les rayons $\mathrm{X}$ sont des photons d'énergie de $\sim 100 \mathrm{eV}$ à $\sim 100 \mathrm{keV}$ ou, de façon équivalente, un rayonnement de longueur d'onde comprise entre $\sim 10$ et $\sim 0,01 \mathrm{~nm}$. Ils sont produits de différentes façons (détaillées dans l'encadré 1) et sont utilisés dans de nombreux domaines scientifiques et techniques (voir encadré 2, p. 12). Une source de lumière est principalement caractérisée par sa brillance : c'est le nombre de photons émis par seconde, par unité d'aire de la source, par unité d'angle solide et dans une largeur de bande d'énergie (bw) de $0,1 \%$. La brillance quantifie l'intensité d'une source, en tenant compte de sa pureté spectrale et de son ouverture angulaire.

Les plus performantes des sources conventionnelles et compactes ont une brillance de $10^{8}-10^{10} \mathrm{ph} / \mathrm{s} / \mathrm{mm}^{2} / \mathrm{mrad}^{2} / 0,1 \%$ bw, à une énergie de rayons $\mathrm{X}$ fixe et limitée aux pics de fluorescence de l'anode; elles ne dépassent pas $10^{4} \mathrm{ph} / \mathrm{s} / \mathrm{mm}^{2} / \mathrm{mrad}^{2} / 0,1 \%$ bw au-dessus de $60 \mathrm{keV}$ (fig. 1) et, pour une large classe d'applications, le trop faible flux nécessite un temps d'exposition prohibitif. Les sources de rayonnement synchrotron sont les plus performantes en termes de brillance [1] ; elles peuvent fournir jusqu'à $10^{21} \mathrm{ph} / \mathrm{s} / \mathrm{mm}^{2} / \mathrm{mrad}^{2} / 0,1 \%$ bw (fig. 1) et sont actuellement les seules machines disponibles pour réaliser les analyses et les recherches les plus ambitieuses nécessitant des X dans la gamme 10-100 keV. Mais les synchrotrons sont de grandes installations dont l'accès est limité (compte tenu du grand nombre d'utilisateurs potentiels), et qui présentent de fortes contraintes d'utilisation (comme, par exemple, le coût et/ou le danger liés à la nécessité de transporter une œuvre d'art ou des cristaux refroidis à l'azote liquide).

Les sources Compton compactes (SCC) sont des machines de taille assez modeste $\left(\sim 100 \mathrm{~m}^{2}\right)$ qui visent une forte brillance

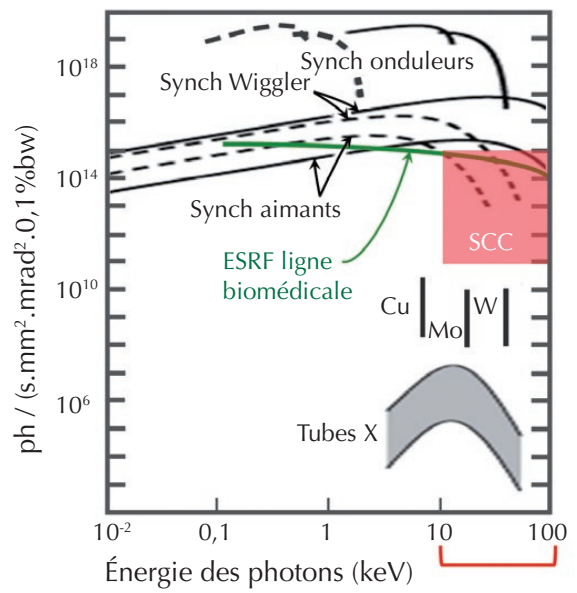

1. Brillances typiques des différentes sources de rayons $X$ : synchrotrons (la ligne biomédicale de l'ESRF est en vert), sources conventionnelles (tubes $X$ à anodes de (u, Mo, W) et projets de sources Compton compactes (SCC, en rose).

( $10^{11}-10^{12} \mathrm{ph} / \mathrm{s} / \mathrm{mm}^{2} / \mathrm{mrad}^{2} / 0,1 \%$ bw et jusqu'à $10^{15}$ pour les projets utilisant un accélérateur linéaire supraconducteur), accordables en énergie dans la gamme 20-100 keV. De nombreuses expériences effectuées aujourd'hui uniquement auprès de synchrotrons pourront ainsi être réalisées au sein d'un laboratoire, d'un musée ou d'un hôpital.

\section{Sources Compton compactes}

Dans une diffusion Compton inverse (fig. 2), un électron d'énergie $\mathrm{E}_{\mathrm{e}}$ cède une fraction de son énergie à un photon de faible énergie $\mathrm{E}_{\mathrm{L}}$ qui, dans le référentiel du laboratoire, est rétrodiffusé avec l'énergie : $\mathrm{E}_{\mathrm{X}}=2 \gamma^{2} \mathrm{E}_{\mathrm{L}}\left(1+\cos \theta_{\mathrm{c}}\right) /\left(1+\gamma^{2} \theta^{2}\right)$, $\left(\gamma>>1\right.$ et $\left.\mathrm{E}_{\mathrm{L}}<<\mathrm{m}_{\mathrm{e}} \mathrm{c}^{2}\right) \quad$ (1), où $\gamma=\mathrm{E}_{\mathrm{e}} / \mathrm{m}_{\mathrm{e}} \mathrm{c}^{2}$ est le facteur de Lorentz de l'électron, $\mathrm{m}_{\mathrm{e}}$ sa masse, $\theta_{\mathrm{c}}$ et $\theta$ sont respectivement l'angle d'incidence et l'angle de diffusion par rapport à la direction de l'électron incident. 


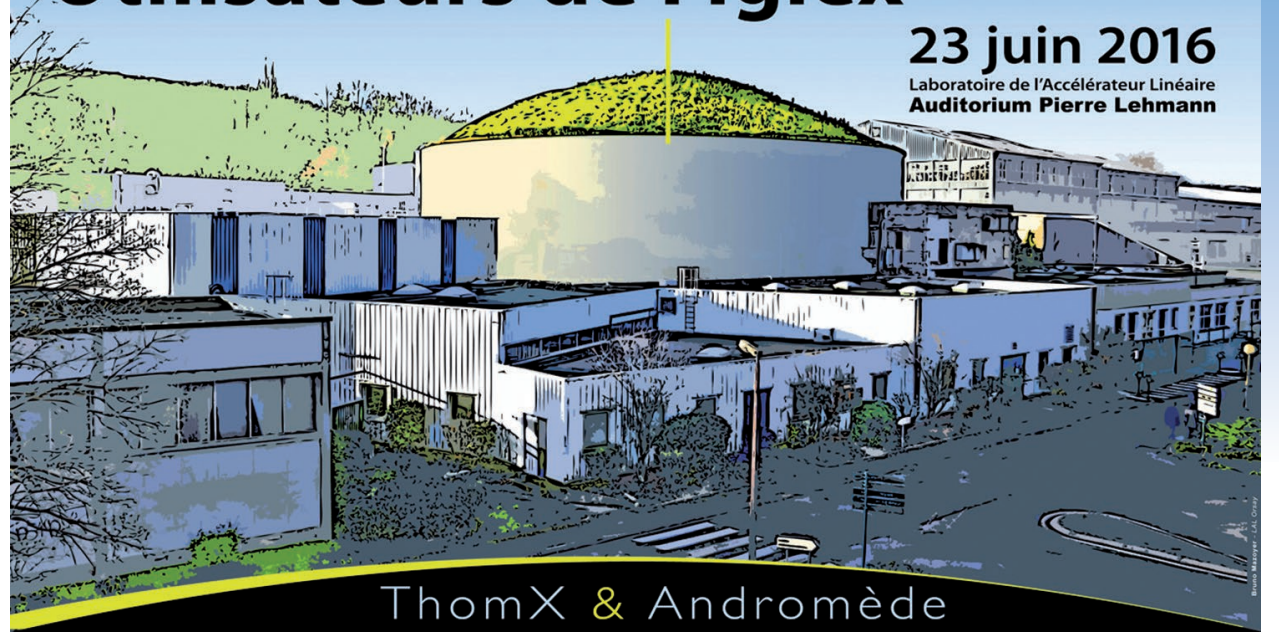

L'équation (1) implique une dépendance univoque entre l'énergie $E_{X}$ d'un photon rétrodiffusé et son angle de diffusion $\theta$ : les rayons $\mathrm{X}$ présentent une distribution en forme de cônes concentriques, où l'énergie diminue lorsqu'on s'éloigne du centre. Ceux produits vers l'avant $(\theta=0)$ ont donc le maximum d'énergie. Ainsi, par un simple diaphragme placé sur l'axe du cône Compton, une bande d'énergie peut être sélectionnée. La figure 3 illustre cette corrélation.

Avec les progrès des lasers, des cavités optiques et des accélérateurs, il est maintenant possible de produire des flux de rayons $\mathrm{X}$ supérieurs à $10^{12} \mathrm{ph} / \mathrm{s}$ à condition que la fréquence de répétition $f_{\text {rep }}$ soit suffisamment élevée, c'est-à-dire dans la gamme 10-100 MHz. Donnons des ordres de grandeur : $10^{13} \mathrm{ph} / \mathrm{s}$ sont obtenus pour $\mathrm{f}_{\text {rep }}=20 \mathrm{MHz}$, un paquet d'électrons de $1 \mathrm{nC}$, un paquet de photons de $10 \mathrm{~mJ}$, et une taille transverse des paquets d'électrons et de photons au point d'interaction (IP) de l'ordre de $40 \mu \mathrm{m}$.

\〉
Affiche de la conférence du 23 juin 2016 où ont été présentés l'état d'avancement et les projets scientifiques des instruments ThomX et Andromède, qui sont en cours d'installation dans l'« Igloo » de l'Université d'Orsay et qui constitueront le centre pluridisciplinaire IGLEX.

\section{Génération des rayons $\mathrm{X}$} encadré 1

Les rayons $\mathrm{X}$ dans la gamme d'énergie $100 \mathrm{eV}$ à $10 \mathrm{keV}$ sont qualifiés de "mous ", ceux de haute énergie ( 10 à $100 \mathrm{keV}$ ) sont dits « durs ». Ils peuvent être générés de trois façons différentes :

- Les « tubes à rayons $X$ » sont des sources conventionnelles, où des électrons accélérés par une différence de potentiel de 10 à 150 kV frappent une anode métallique (en tungstène, cuivre ou molybdène). Du freinage des électrons résulte un spectre continu de photons jusqu'aux rayons $X$ durs, auquel se superposent des raies de fluorescence spécifiques de l'anode utilisée. Le freinage des électrons produit un dégagement important de chaleur, qui limite le flux de ce type de source.

- En stockant des électrons dans un accélérateur circulaire, la courbure des orbites entraîne un rayonnement dit synchrotron. Des électrons de quelques GeV émettent des photons dans le domaine des $X$. Les sources de rayonnement de troisième génération (SOLEIL, ESRF...) utilisent principalement le rayonnement produit par des éléments spécifiques appelés onduleurs.

- Lorsqu'on envoie un faisceau laser classique (photons de $\sim 1 \mathrm{eV}$ ) sur des électrons ultrarelativistes, ce choc produit des photons rétrodiffusés de haute énergie. C'est l'effet Compton inverse. On obtient ainsi des rayons $\mathrm{X}$ durs de quelques dizaines de keV, avec un laser à $1 \mathrm{eV}$ et des électrons de quelques dizaines de $\mathrm{MeV}$ (c'est-à-dire environ 100 fois moins énergétiques que ceux utilisés pour produire le rayonnement synchrotron). Cette diffusion Compton permet donc de concevoir des sources de rayons $\mathrm{X}$ compactes (installations de $\sim 100 \mathrm{~m}^{2}$ ), qui sont l'objet du présent article.

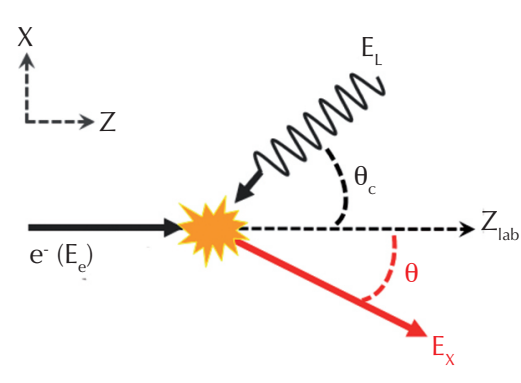

2. Schéma du processus de diffusion Compton inverse.
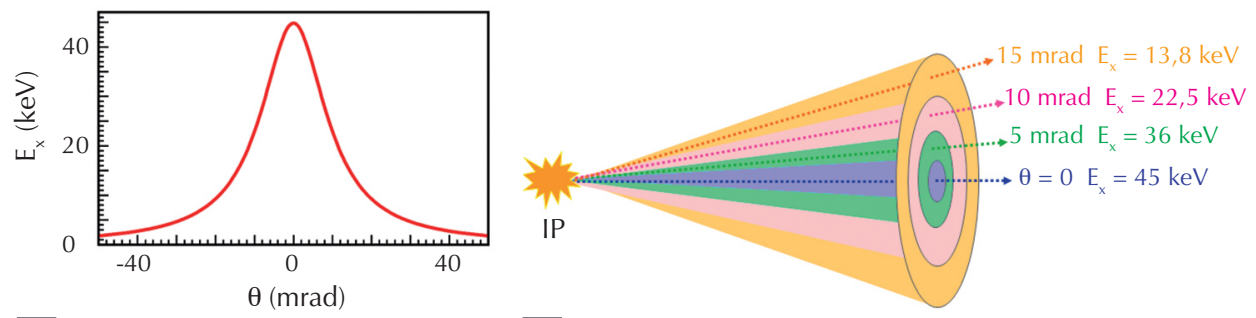

$\mathrm{b}$

3. Répartition en énergie et en angle des photons Compton rétrodiffusés. (a) Énergie des photons Compton en fonction de leur angle de diffusion pour des collisions frontales d'impulsions laser infrarouge de longueur d'onde $1,06 \mu \mathrm{m}\left(E_{L}=1,17 \mathrm{eV}\right)$ avec des paquets d'électrons de $50 \mathrm{MeV}(\gamma \sim 100)$. (b) Illustration schématique des cônes concentriques du faisceau Compton. IP est le point d'interaction électron/photon laser. 


\section{Source utilisant un anneau de stockage}

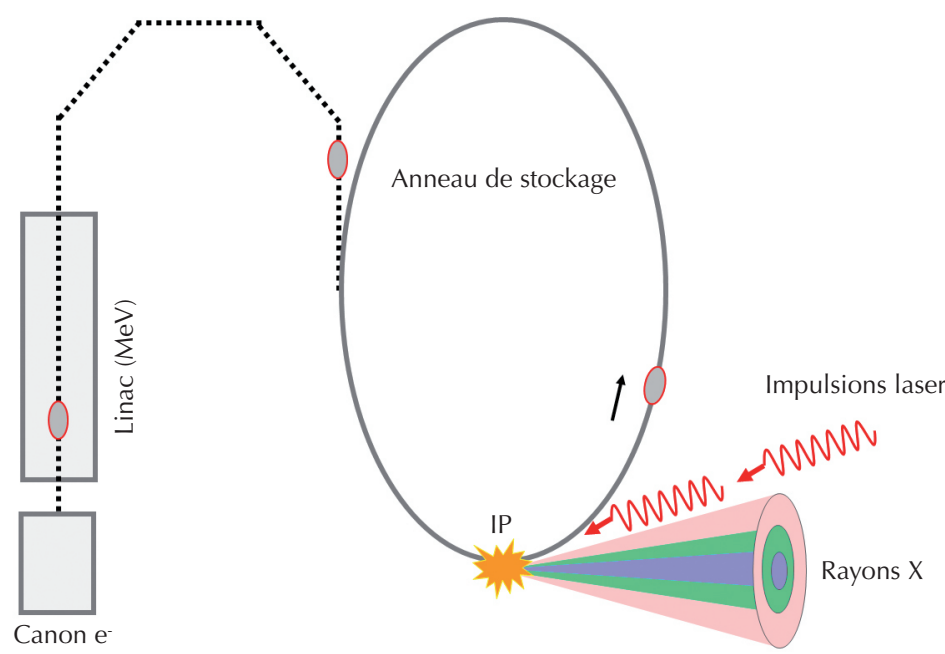

Source utilisant un Linac seul

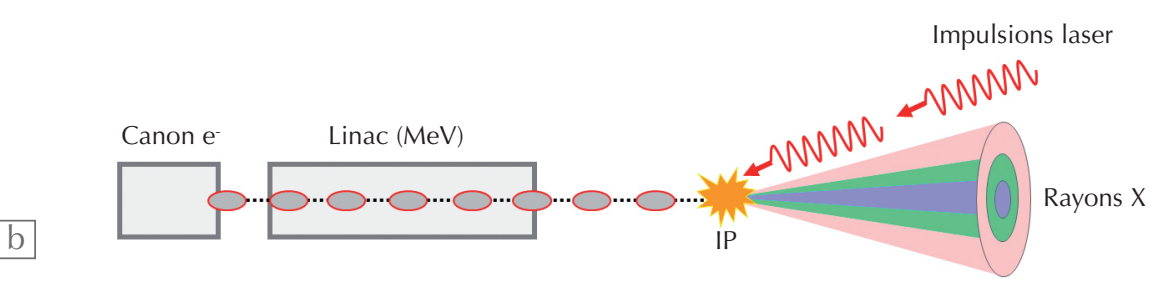

4. Schémas de principe des SCC de haut flux avec et sans anneau de stockage, respectivement (a) et (b). (Linac = accélérateur linéaire)

\section{Utilisation des rayons $\mathbf{X}$}

encadré 2

- La gamme de longueurs d'onde des rayons X s'étendant de la dimension d'un atome $\left(\sim 0,1 \mathrm{~nm}\right.$ ) à celle $\mathrm{d}^{\prime}$ une molécule (quelques $\mathrm{nm}$ ), la diffraction des rayons $X$ permet l'étude de la structure de très nombreux composés chimiques et de matériaux totalement ou partiellement cristallisés. Par ailleurs, l'énergie élevée des rayons X conduit à l'ionisation des matériaux irradiés, générant de la fluorescence $X$ utilisée pour des analyses chimiques.

- Les $X$ durs traversent facilement la matière peu dense, constituée d'éléments légers (nombre atomique Z faible), tandis qu'ils sont absorbés par la matière dense. Cette propriété permet l'imagerie par contraste d'absorption (la radiographie et la tomographie habituelles).

- L'absorption des rayons X par la matière biologique est faible et constitue une limitation de la radiographie. Pour améliorer les images, la réfraction, la diffraction et les interférences de rayons $X$ sont utilisées dans I'imagerie par contraste de phase. Une meilleure visibilité des frontières entre des domaines présents dans un échantillon est ainsi obtenue en exploitant la phase de I'onde X. Pour cela, la source doit être transversalement quasi cohérente. Cette technique utilise les différences de phase résultant des variations spatiales de l'indice de réfraction au sein de l'échantillon. Comme le rayonnement se propage au-delà de l'échantillon, les portions de la surface d'onde qui se sont propagées de part et $d^{\prime}$ autre $d^{\prime}$ une frontière interne de l'échantillon produisent une série de franges par diffraction de Fresnel.

- La radiothérapie est une autre grande utilisation du rayonnement X. Les cellules tumorales se divisant plus rapidement que les cellules saines, sont plus sensibles aux rayonnements ionisants, et il est possible d'éliminer une tumeur en la soumettant à une forte dose de rayonnement.

\section{>>}

Deux conceptions de machines fondées sur la rétrodiffusion Compton permettent d'atteindre un taux de répétition élevé, selon que les photons laser entrent en collision avec des électrons provenant d'un petit anneau de stockage d'une dizaine de mètres de périmètre (fig. 4a), ou avec ceux d'un accélérateur linéaire (Linac) supraconducteur (fig. 4b) - l'une et l'autre présentant des avantages et des difficultés techniques spécifiques.

Dans le cas d'un anneau de stockage, $\mathrm{f}_{\text {rep }}$ est déterminée par la fréquence de révolution des électrons dans l'anneau, permettant d'obtenir une brillance de $10^{11}-10^{12} \mathrm{ph} / \mathrm{s} / \mathrm{mm}^{2} / \mathrm{mrad}^{2} / 0,1 \%$ bw (bas du rectangle rose de la figure 1) et un flux de $10^{12}-10^{13} \mathrm{ph} / \mathrm{s}$.

Dans le schéma utilisant un accélérateur linéaire sans anneau de stockage, la fréquence $\mathrm{f}_{\text {rep }}$ est déterminée par la fréquence de la source d'électrons qui doit nécessairement être un dispositif supraconducteur. Bien que des projets d'injecteur d'électrons de haute performance soient actuellement en développement [2], la technologie supraconductrice dans ce domaine n'est pas encore assez mature pour atteindre à la fois les cadences et le nombre d'électrons requis.

Dans les deux schémas, les impulsions d'un laser de faible puissance moyenne, de fréquence $f_{\text {rep }}$, sont amplifiées puis accumulées et stockées dans une cavité optique du type Fabry-Perot de finesse très élevée. (La finesse est le nombre moyen d'allers-retours qu'effectue une impulsion réfléchie dans la cavité.)

Actuellement, une seule SCC, construite par Lyncean Technologies, fonctionne dans le monde (depuis 2008). Elle utilise un anneau de stockage. La brillance de cette source est de l'ordre de $\sim 10^{8} \mathrm{ph} / \mathrm{s} / \mathrm{mm}^{2} / \mathrm{mrad}^{2} / 0,1 \%$ bw, et $\sim 10^{10} \mathrm{ph} / \mathrm{s}$ sont produits à une énergie maximum d'une trentaine de $\mathrm{keV}$ [3]. Plusieurs sources Compton d'intensité, de brillance et d'énergie de rayons $\mathrm{X}$ plus élevées sont en projet dans le monde [4]. Les paquets d'électrons de $0,1-1 \mathrm{nC}$ à un taux de répétition de quelques dizaines de $\mathrm{MHz}$ exigés pour produire des flux supérieurs à $10^{12} \mathrm{ph} / \mathrm{s}$ ne sont pour l'instant disponibles qu'avec des anneaux de stockage. Parmi ces projets, ThomX [5] est le plus avancé et vise à produire un flux de $\sim 10^{12}-10^{13} \mathrm{ph} / \mathrm{s}$, avec une brillance de $\sim 10^{11}-10^{12} \mathrm{ph} / \mathrm{s} / \mathrm{mm}^{2} / \mathrm{mrad}^{2} / 0,1 \%$ bw. 


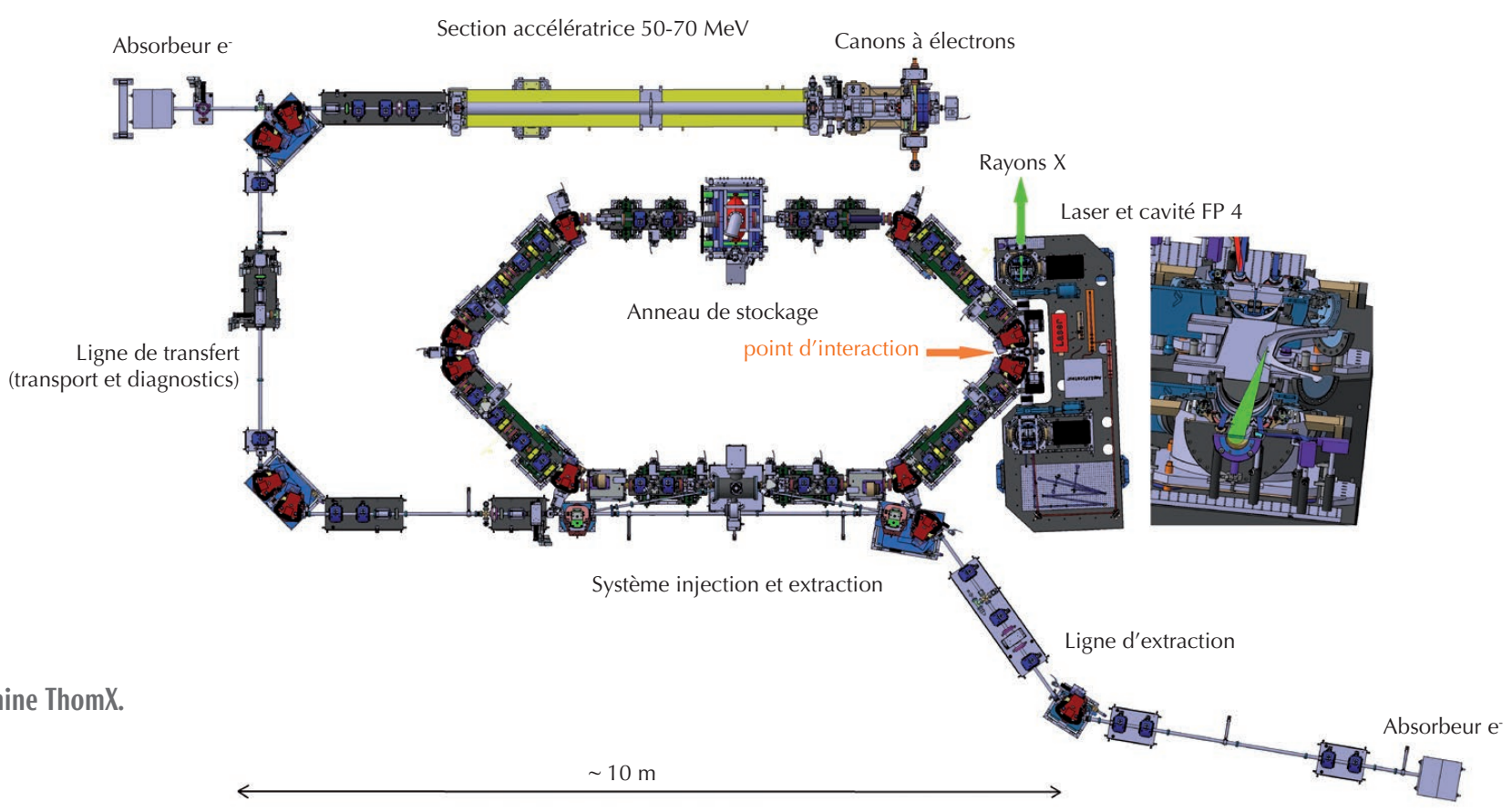

5. La machine ThomX.

\section{La machine ThomX}

La source et le stockage des électrons

Pour ThomX (fig. 5), des électrons d'une énergie de quelques eV sont d'abord photoémis par l'impact d'une impulsion laser sur une cathode; puis ils se propagent dans un accélérateur linéaire qui porte leur énergie à 50-70 MeV. Une ligne de transfert adapte le faisceau d'électrons pour les stocker dans un petit anneau de $18 \mathrm{~m}$ de circonférence. Afin de maximiser le flux de rayons X, la charge du paquet est portée à $1 \mathrm{nC}$ (6,2 $10^{9}$ électrons) en faisant varier l'énergie laser, et sa taille transverse est réduite au niveau du point d'interaction (tableau 1). À chaque tour d'anneau, l'aire transverse et la longueur du paquet d'électrons augmentent par suite d'un échauffement, dû aux collisions Compton, et de la diffusion coulombienne multiple au sein du paquet. Des systèmes de contre-réaction très rapides sont prévus pour lutter contre les instabilités (d'énergie, de longueur et d'émittance du paquet) causées par des perturbations électromagnétiques dans l'anneau et pouvant conduire à la perte du paquet. Par exemple, la position du faisceau est mesurée à chaque tour et corrigée. Au bout de $20 \mathrm{~ms}$, la structure du paquet est néanmoins dégradée. Il est alors éjecté vers un blindage qui l'absorbe, et est remplacé par un nouveau paquet.

La source de photons laser et leur stockage dans une cavité optique

Le système optique de ThomX est schématisé sur la figure 6. Pour obtenir des
6. Vue schématique du système optique de ThomX comprenant l'oscillateur laser, l'amplificateur fibré, la cavité constituée de quatre miroirs (résonateur optique) et le système de stabilisation de fréquence. 
\〉

flux de photons exploitables, il faut amplifier la puissance d'un faisceau laser pulsé [6] et " empiler" ses impulsions. Nous avons choisi d'utiliser un résonateur (aussi nommé "cavité optique »), constitué de deux miroirs plans et de deux miroirs sphériques [7] ayant un facteur de gain $G$ 10000 [8]. Pour atteindre cette grande valeur de $G$, la fréquence $v$ du laser doit être stabilisée avec une précision $\Delta v / v=c /(\pi v G L)$, où $L / 2$ est la longueur de la cavité. Pour ThomX, il faudra atteindre $\Delta v / v \sim 10^{-12}$. En outre, pour atteindre un flux de rayons $X$ de $\sim 10^{13} \mathrm{ph} / \mathrm{s}$, les impulsions laser en amont de la cavité optique doivent être amplifiées avec un système de fibres dopées à l'ytterbium pompées par diodes - développé au CELIA à Bordeaux [7] - qui délivrera une puissance moyenne de $200 \mathrm{~W}$. En positionnant la cavité optique de sorte que le trajet de la lumière croise celui du faisceau d'électrons, nous profiterons de ces deux étages d'amplification pour produire un haut flux de rayons $\mathrm{X}$. Le défi technologique est de faire fonctionner le système optique sans interruption dans l'environnement d'un accélérateur d'électrons [7]. En particulier, nous appliquerons les méthodes de stabilisation de fréquence des oscillateurs laser développés en métrologie en nous assurant que l'accélérateur n'induit aucun bruit de phase ou d'amplitude qui pourrait perturber l'asservissement fréquentiel du faisceau laser. Les paramètres attendus pour le système optique sont présentés dans le tableau 2.

\section{Applications potentielles}

\section{Le faisceau de rayons $\mathrm{X}$}

Le faisceau de rayons $\mathrm{X}$ disponible à la sortie de la cavité Fabry-Perot a une demiouverture de 10 mrad.

Dans une première configuration qui exploite la divergence intrinsèque de l'effet Compton (cf. équation (1)), un large faisceau conique est disponible (haut de la figure 7). Un tel faisceau a une largeur spectrale de quelques pourcents à plusieurs dizaines de pourcents selon l'angle solide sélectionné.

Dans une seconde configuration, seule la partie centrale du faisceau est sélectionnée et focalisée par un dispositif, tel un transfocateur [9], situé le plus près possible du point d'interaction (IP) avant que le faisceau ne diverge trop. Ce faisceau étroit

\begin{tabular}{|l|c|}
\hline Finesse de la cavité optique & $\sim 30000$ \\
\hline Longueur d'onde du rayonnement laser & $1,032 \mu \mathrm{m}$ \\
\hline Énergie de l'impulsion stockée dans le résonateur & 3 à $17 \mathrm{~mJ}$ \\
\hline Puissance moyenne stockée dans le résonateur & 500 à $1000 \mathrm{~kW}$ \\
\hline Longueur de l'impulsion stockée dans le résonateur & $10 \mathrm{ps}$ \\
\hline Largeur spectrale de l'impulsion stockée dans le résonateur & $<2 \mathrm{~nm}$ \\
\hline Dimensions transverses de l'impulsion stockée dans le résonateur à l'IP & $40 \mu \mathrm{m}$ \\
\hline Puissance moyenne du faisceau laser après amplification & 10 à $200 \mathrm{~W}$ \\
\hline Fréquence de répétition des impulsions laser & $35,68 \mathrm{MHz}$ \\
\hline
\end{tabular}

Tableau 2. Paramètres du système optique (laser et résonateur) de ThomX
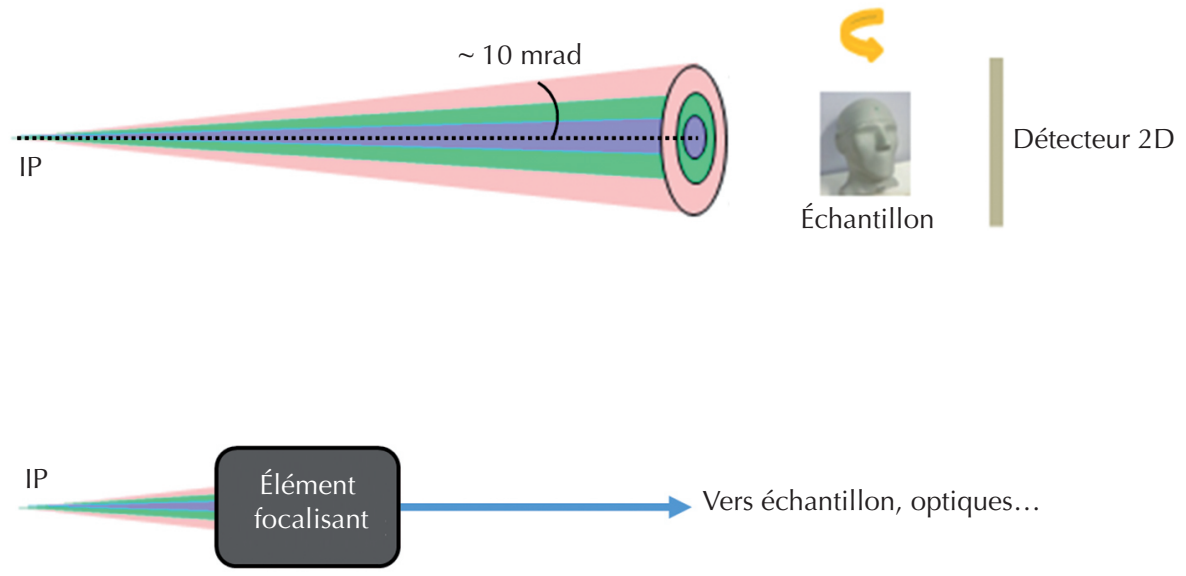

\section{Deux utilisations d'un faisceau Compton.}

En haut, un faisceau étendu et conique (couleur rose). En bas, un faisceau fin et quasi monochromatique.

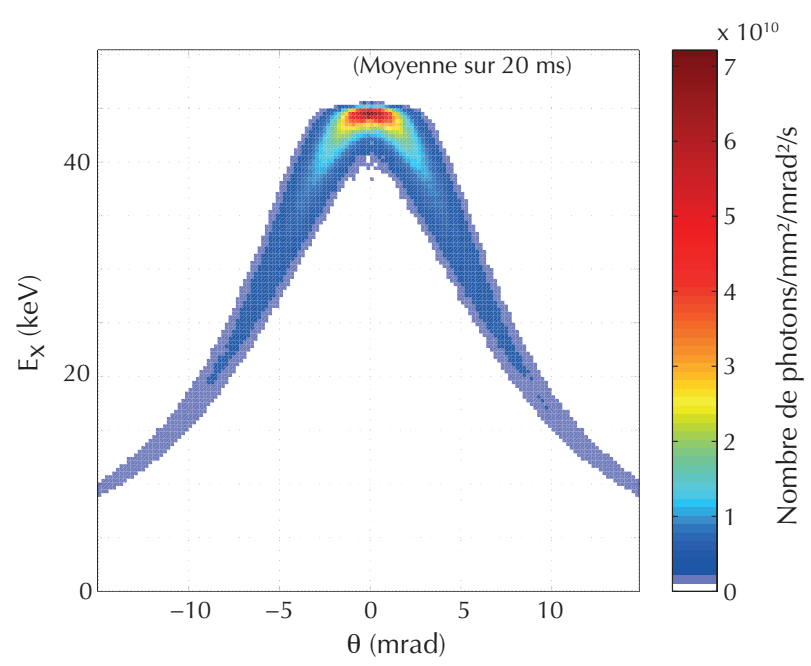

8. Dépendance en angle et en énergie du flux spectral (code de couleurs) attendu pour ThomX. Simulation réalisée avec les paramètres des tableaux 1 et 2 . 
(schématisé en bas de la figure 7), quasimonochromatique, peut ensuite être envoyé dans un dispositif optique supplémentaire (miroir, monochromateur...) avant d'éclairer un échantillon.

Selon les applications, le grand faisceau conique ou, au contraire, le faisceau étroit et quasi monochromatique sera le plus approprié.

\section{Caractérisation et expériences}

La caractérisation du faisceau consistera à mesurer son intensité, sa gamme d'énergie, sa distribution spectrale et angulaire, et son degré de cohérence transverse ${ }^{(a)}$. Les flux spectraux seront déterminés à partir du faisceau divergent en mesurant, en un grand nombre de petits angles solides du spectre d'émission, l'énergie moyenne et le flux. Ces mesures permettront de reconstruire le flux dans l'espace énergie-angle d'émission, dont la simulation réalisée avec les paramètres des tableaux 1 et 2 est présentée sur la figure 8 . Le degré de cohérence transverse sera déterminé à partir d'expériences à doubles fentes d'Young [10], dans lesquelles l'intensité des rayons X sera enregistrée en fonction de leur direction d'émission.

Les diverses caractéristiques du faisceau de ThomX dans les deux configurations de la figure 7 (divergent ou focalisé), ainsi que les principales applications correspondantes, sont présentées dans le tableau 3.
Les caractéristiques du faisceau focalisé du tableau 3, bien qu'inférieures à celles des synchrotrons, seront suffisantes pour mener à bien toute une série d'expériences dans le domaine de la science des matériaux [5] et dans celui de l'héritage culturel [11], en particulier. Le faisceau Compton conique (divergent) permettra, lui, d'illuminer un échantillon de plusieurs $\mathrm{cm}^{2}$ sans avoir à le déplacer ce qui, en imagerie et en thérapie, présente un grand avantage sur d'autres sources pour lesquelles il est nécessaire de scanner l'échantillon en le déplaçant (dans les études cliniques réalisées à l'ESRF par exemple, le patient ou l'échantillon doit être déplacé verticalement car la taille verticale du faisceau est d'environ $2 \mathrm{~mm}$ ). Les flux angulaires et spectraux (voir le tableau 3) permettront de faire toute une série d'études réalisables actuellement uniquement auprès des sources synchrotron. Par exemple, la dose typique délivrée pour une imagerie conventionnelle crânienne sur la ligne biomédicale de l'ESRF (ID17) est de $2,4 \mathrm{~Gy} / \mathrm{s} / \mathrm{cm}^{2}$, à comparer à $2,0 \mathrm{~Gy} / \mathrm{s} / \mathrm{cm}^{2}$ attendu avec le faisceau de ThomX [5]. Autre exemple : pour la radiothérapie, les $210^{12} \mathrm{ph} / \mathrm{s}$ délivrés par ThomX dans un disque de $5 \mathrm{~cm}$ de diamètre correspondent à $10^{9} \mathrm{ph} / \mathrm{s} / \mathrm{mm}^{2}$, du même ordre de grandeur que l'intensité moyenne utilisée dans les essais cliniques de radiothérapie en cours à l'ESRF [12].

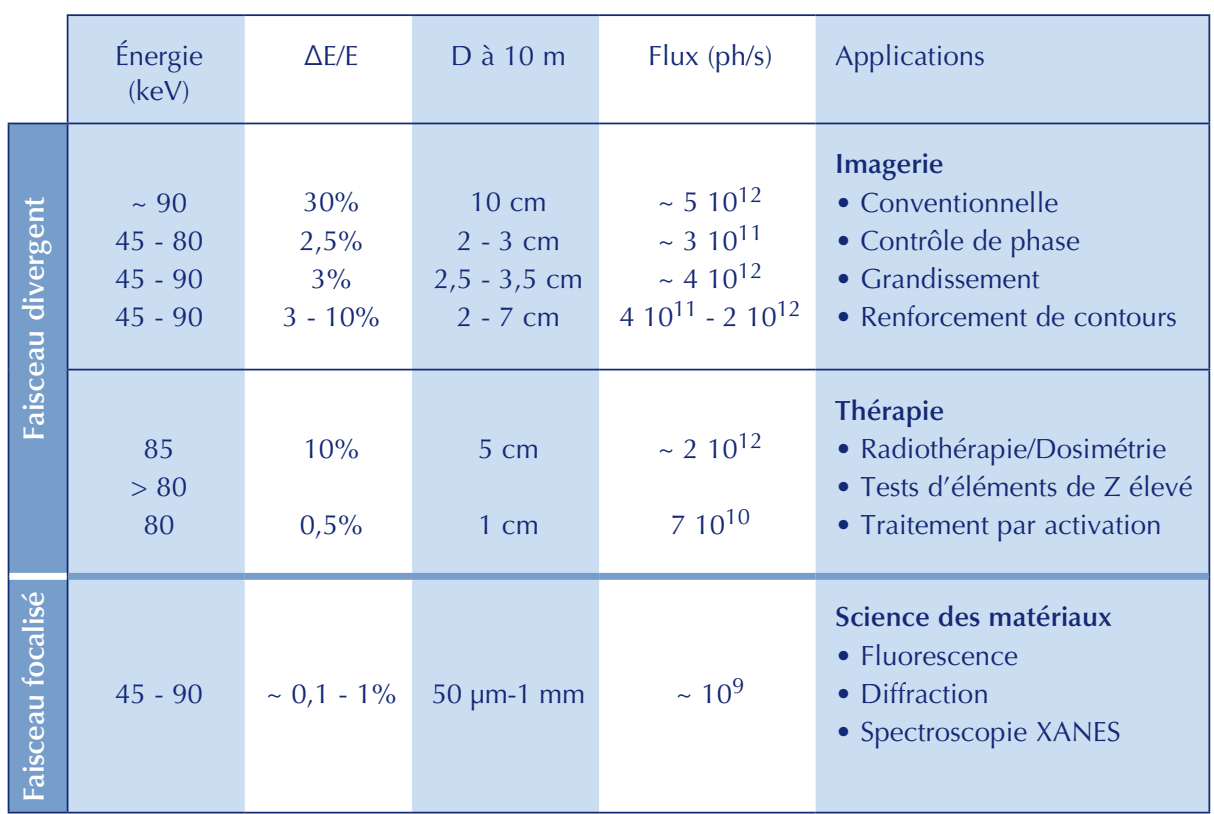

Tableau 3. Principales caractéristiques et applications des faisceaux X issus de ThomX. $\Delta E / E$ est la dispersion relative en énergie, D le diamètre du faisceau à $10 \mathrm{~m}$ du point d'interaction (IP). Les flux indiqués ont été calculés au niveau de l'échantillon sur la base d'un flux total (avant sélection optique) de $10^{13} \mathrm{ph} / \mathrm{s}$.

\section{Conclusion et planning}

La collaboration en charge du projet ThomX est multidisciplinaire. Elle vise à construire un démonstrateur de source X compacte à la pointe de la technologie et à établir ses performances dans le domaine médical, dans l'étude du patrimoine culturel (restauration d'œuvres d'art, contrefaçon, histoire d'une ouvre) et de la science des matériaux, avec des extensions possibles, par exemple dans le domaine de la sécurité (aéroports, douanes...) ou dans l'industrie (identification de défauts de fabrication sans détruire le matériau...).

Les différentes études et simulations sont décrites dans le Technical Design Report publié en 2014 [5]. L'intégration de ThomX se fera dans le bâtiment appelé "l'Igloo » sur le campus d'Orsay (voir l'affiche p. 15). Les travaux d'infrastructure du bâtiment, démarrés début 2016, devraient durer un peu moins d'un an. Durant cette période, une intégration partielle sera effectuée (pré-montage et tests des éléments de la machine) en attendant l'intégration finale dans les locaux prévue fin 2016. Après l'installation, la mise en route de ThomX permettra d'obtenir un premier faisceau de rayons X en 2017.

(a) La cohérence transverse d'une source est sa capacité à envoyer des ondes spatialement cohérentes en un point donné.

\section{Références}

1 M.E. Couprie et al., Reflets de la Physique, 34-35 (2013) 18-23.

2• A. Arnold et al., Phys. Rev. ST Accel. Beams 14 (2011) 024801.

3• M. Bech et al., J. Sync. Rad. 16 (2009) 43.

4• M. Jacquet, Nucl. Instrum. Methods B 331 (2014) 1.

5• A. Variola et al., ThomX Technical Design Report (2014) < in2p3-00971281 >

6• L. Casano et al., Laser and Unconv. Opt. Jour. 55 (1975) 3.

7• J. Bonis et al., JINST 7 (2012) P01017.

8• A. Borzsonyi et al., Appl. Opt. 52 (2013) 8376

9• A. Snigirev et al., Nature 334 (1996) 49.

10• D. Paterson et al., Opt. Com. 195 (2001) 79.

11• P. Walter et al., C.R. Physique 10 (2009) 676.

12• M. Jacquet et al., Phys. Med. 31 (2015) 596.

Remerciements

Les auteurs tiennent à remercier l'ensemble des personnes engagées sur ThomX, ingénieurs, chercheurs, techniciens et administratifs, et veulent souligner les contributions d'Alessandro Variola (initiateur du projet) et de Jacques Haissinski pour son soutien sans faille. Ce travail bénéficie d'une aide de l'État gérée par l'Agence Nationale de la Recherche au titre du programme Investissements d'avenir (référence ANR1O-EQPX-51), de la région Île-de-France et du CNRS. 